\title{
Tagungen/Congrès
}

Training im Umgang mit Agoraphobie oder Helfen lernen bei Paniksyndromen

Seminar der SV-Sozjalberatung,

9. bis 13. März 1987 in Zürich

Information und Anmeldung:

SV-Sozialsekretariat, SV-Service

Neumünsterallee 1, 8032 Zürich

Telefon 012518252

Anmeldeschluss 2, Februar 1987

Psychische Gesundheit und Krankheit

im Alter

Fachtagung der PRO SENECTUTE und PRO MENTE SANA

Journées d'étude vieillesse et santé mentale Bern 19./20. März 1987

Anmeldungen bis zum 16. Februar 1987 an: Pro Senectute Schweiz, Personalschulung. Postfach

8027 Zürich

\section{International Healih}

in an Era of Economic Constraint:

The Challenge.

Fifth International Congress,

World Federation of Public Health

Associations.

22. -27. März 1987 in Mexico-City, Mexiko Information: $V$. International Congress

Organizing Committee

Leibnitz 32-1st Floor, 11590 Mexico. D.F

Mexico

IX Congreso International de Medicina e Higiene Escolar y Universitaria

27. -30. Mai 1987, Madrid

Scientific information: Apartado de Correos 10.017. Madrid (España)
Congrès de Pneumologie de langue française

11./12./13, Juni, Grenoble

Organisation secrétariat: Pavillon D2

CHRG BP $216 \mathrm{X}$

F-38043 Grenoble Cedex

Generalversammlung der VESKA/

Assemblée générale de la VESKA

17. Juni 1987, Fribourg

Schweizerischer Krankenhauskongress Congrès suisse des hôpitaux

20. bis 22. Oktober 1987, Interlaken

Voranzeige/Date à retenir:

Wissenschaftliche Arbeitstagung

der Schweizerischen Gesellschaft für Sozialund Präventivmedizin/Journées

scientifiques

de la societé suisse de médecine sociale et préventive

25./26. Juni 1987 in Interlaken

Information/Information: Abt. für Sozialund Präventivmedizin der Universität Basel St. Albanvorstadt 19, 4052 Basel

Le médecin généraliste hier, aujourd'hui et demain

Jahreskongress der Schweizerischen

Gesellschaft für Allgemeinmedizin,

1./2./3. Oktober 1987
Voranzeige/First announcement: Conference

on International Travel Medicine 5. -8. April 1988, Zürich

Further informations: Conference on International Travel Medicine Interconvention $A G$, c/o Swissair CH-8058 Zürich-Airport, Switzerland

Symposium on Health planning in rheumatology with special emphasis on perspective, models of cure and care. Vom 23. bis 26. März 1987

Organisiert durch die Gesellschaft der Rheumatologie der DDR, in Dresden

Information:

DDR-8010 Dresden, Friedrichstrasse 41, Bezirkskrankenhaus, I. Medizinische Klinik.

\section{Santé et environnement}

Cours de perfectionnement interdisciplinaire -12 jeudis durant l'hiver 1986/1987

Organisé par:

- l'Institut du Génie de l'Environnement (EPFL),

- l'Institut Universitaire de Médecine Sociale et Préventive, de Lausanne,

- avec la collaboration dautres institutions universitaires et publiques.

Information:

Institut du Génie de l'Environnement, EPFL, 1015 Lausanne. 